Revista Brasileira de Agricultura Irrigada v.6, no. 2, p. 97-105, 2012

ISSN 1982-7679 (On-line)

Fortaleza, CE, INOVAGRI - http://www.inovagri.org.br

DOI: $10.7127 /$ rbai.v6n200135

Protocolo 135 - 23/02/2012 Aprovado em 17/04/2012

\title{
PRODUTIVIDADE DA CANA-DE-AÇÚCAR RB83-2847 IRRIGADA EM PARANAVAÍ, PARANÁ
}

Allan Cunha Barros ${ }^{1}$, Rubens Duarte Coelho², Fábio Ricardo Marin³ ${ }^{3}$ Pedro Robinson Fernandes de Medeiros ${ }^{4}$, Antenor de Oliveira Aguiar Netto ${ }^{5}$ e Dorothee Luize Polzer ${ }^{6}$

\section{RESUMO}

A disponibilidade hídrica no solo favorece uma maior produção da cultura da cana, assim, a decisão de irrigar torna-se decisiva para obter altas produções, mas para que essa opção seja rentável o aumento de produção de açúcar por cana, em função da irrigação, deve compensar o investimento com o sistema de irrigação. A utilização de modelos permite que sejam estimados dados para a tomada de decisão Assim, o objetivo do trabalho foi gerar informações sobre a produtividade potencial da cana-de-açúcar, cultivar RB83-2847, na cidade de Paranavaí - PI, com plantio e colheita em diferentes datas, através de estimativas de crescimento da cultura utilizando o CANEGRO/DSSAT.Os cenários foram baseados em 4 datas de plantio ( 15 de janeiro, 15 de março, 15 de setembro e 15 de novembro) x 2 épocas de colheita ( 1 e 1,5 anos) x 2 sistemas (irrigado e sequeiro), totalizando 16 cenários por cidade. Assim, foi possível verificar a produtividade atingida para a cultivar RB83-2847 em

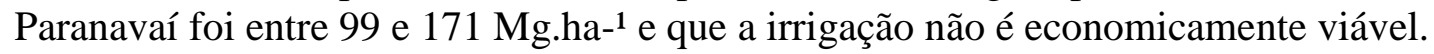

Palavra-chave: Dssat, simulação de crescimento, irrigação em cana-de-açúcar.

\section{PRODUCTIVITY OF SUGARCANE RB83-2847 IRRIGATED IN PARANAVAÍ, PARANÁ}

\footnotetext{
ABSTRACT

The soil water availability favors greater production of sugar cane cultivation, so the decision to irrigate becomes critical for high yields, but that this option is profitable to increase production of sugar cane, depending on irrigation must compensate for the investment with the irrigation system. The use of models allows to estimate data for decision making. The aim of this study was to generate information on the potential productivity of

${ }^{1}$ Prof. Dr. Irrigação e Drenagem,UFAL - Arapiraca, CEP57309-005, Arapiraca, AL Fone (82)3482 1831. email: allan-cunha@hotmail.com

2 Prof. Doutor, Depto de Engenharia de Biossistemas, ESALQ/USP, Piracicaba, SP.

${ }^{3}$ Pesquisador, EMBRAPA, Campinas, SP.

${ }^{4}$ Pós-Doutorando, UFRPE, Recife, PE.

${ }^{5}$ Prof. Doutor, Depto de Engenharia Agronômica, UFS, São Cristóvão, SE.

${ }^{6}$ Graduada, PUC-Campinas, Campinas, SP.
} 


\section{PARANÁ}

sugarcane, cultivar RB83-2847, the city of Paranavaí - PI, with planting and harvesting on different dates, by estimating crop growth using CANEGRO / DSSAT. The scenarios were based on four planting dates (January 15, March 15, September 15 and November 15) x 2 harvest times ( 1 and 1.5 years) x 2 systems (irrigated and rain), totaling 16 scenarios for the city. Thus it was possible to verify the productivity achieved by the cultivar in Paranavaí RB83-2847 was between 99 and 171-Mg.ha ${ }^{1}$ and that irrigation is not economically viable.

Keyword: DSSAT, growth simulation, irrigation in sugarcane.

\section{INTRODUÇÃO}

O Brasil, por possuir duas estações distintas durante o ano, é o único país no mundo com duas colheitas anuais: uma do Norte - Nordeste, que começa em setembro e continua até abril, e a outra na região Centro - Sul, que vai de maio a dezembro (SCARPARI, 2007), o que o torna o maior produtor mundial (501.536 mil toneladas métricas), possuindo maior área cultivada (6.290 mil ha) no mundo (AGRIANUAL, 2009).

A cana-de-açúcar é cultivada no Brasil desde o século XVI e expandiu-se no país, sendo utilizada na produção de açúcar para o consumo interno e exportação, gerando divisas para o país, álcool, representando uma alternativa na substituição do combustível derivado do petróleo. Também serve como um recurso forrageiro na alimentação suplementar dos ruminantes no período de estiagem (RAMOS, 2006) e ainda como matéria prima para a fabricação de rapadura, melado e aguardente. Seus resíduos também possuem grande importância econômica: o vinhoto é transformado em adubo e o bagaço, em combustível (CAPUTO et al., 2008).

No Estado do Paraná, a cultura ocupa uma área de 380 mil hectares, com uma produção anual de 31 milhões de toneladas de cana-de-açúcar. Para o Estado, estima-se um aumento de 150.000 ha na área plantada com a cultura para os próximos anos. Com este aumento o estado chegará a cultivar a cana-de-açúcar em uma área de aproximadamente de 530.000 ha (Oliveira et al., 2008).

Já é fato que a disponibilidade hídrica no solo favorece uma maior produção da cultura da cana, assim, a decisão de irrigar torna-se decisiva para obter altas produções, mas para que essa opção seja rentável o aumento de produção de açúcar por cana, em função da irrigação, deve compensar o investimento com o sistema de irrigação, com a energia que será utilizada e com o custo da água (dependendo da região) (Barros et al. 2012).

A região Sul do país possui boa distribuição de chuva durante o ano, levantando-se o questionamento da necessidade da necessidade em se utilizar a irrigação com técnica para o aumento de produtividade. Para a isso a forma mais ideal de responder tal questionamento é através da realização de pesquisas de campo. No entanto, a realização de pesquisas de campo para determinar a viabilidade da irrigação são onerosas, já que a cana é um cultura semiperene e deve ser cultivada por 5 ou mais anos.

Uma alternativa a realização de experimentos em campo é a utilização do modelos de simulação, estes permitem a quantificação da contribuição de vários processos fisiológicos e elementos climáticos. Eles são ferramentas poderosas que aumentam a eficiência da pesquisa, permitindo a análise da performance das culturas em diferentes condições climáticas (CHEROO-NAYAMUTH, 2000).

Existem diversos modelos para simulação de produção de cana-de-açúcar, mas segundo Godoy (2007), os dois principais correntemente em uso mundialmente são o APSIM (Agricultural Production System sIMulator)- sugarcane, modelo desenvolvido pela unidade de pesquisa de sistemas de produção agrícola CSIRO (Commonwealth Scientific and Industrial Research Organisation) 
PRODUTIVIDADE DA CANA-DE-AÇÚCAR RB83-2847 IRRIGADA EM PARANAVAÍ, PARANÁ

(O'LEARY et al., 1999) e o CANEGRO (sugarCANE GROwth model) desenvolvido pela associação sul africana do açúcar (INMAN-BAMBER, 1995).

Assim, o objetivo do trabalho foi gerar informações sobre a produtividade potencial da cana-de-açúcar cultivar RB832847 na cidade de Paranavaí - PI, com plantio e colheita em diferentes datas, através de estimativas de crescimento da cultura utilizando o CANEGRO/DSSAT.

\section{METODOLOGIA}

$\mathrm{O}$ estudo foi desenvolvimento em parceria entre a ESALQ/USP e a Embrapa Informática Agropecuária. As simulações de crescimento de cana-de-açúcar foram para Paranavaí-PR (latitude $23^{\circ} 04^{\prime} \mathrm{S}$ e longitude $52^{\circ} 25^{\prime}$ O), utilizando-se um modelo de simulação CANEGRO, que faz parte do pacote de modelos no sistema computacional DSSAT (Decision Support System for Agrotechnology Transfer).

O solo utilizado nas simulações foi o Latossolo Vermelho Escuro, cujas características físicas foram retiradas de Marun (1996).

Tabela 1: Características Físicas dos solos utilizados para cada região

\begin{tabular}{ccccc}
\hline Profundidade (m) & Argila & Silte & Areia & $\begin{array}{c}\text { Densidade do solo } \\
\left(\text { kg.dm- }{ }^{3}\right)\end{array}$ \\
\cline { 2 - 4 } & & $(\%)$ & & - \\
0,05 & 9,0 & 1,0 & 90,0 & - \\
$0,05-0,15$ & 11,0 & 2,0 & 87,0 & - \\
$0,15-0,25$ & 14,0 & 1,0 & 85,0 & - \\
$0,25-0,35$ & 16,0 & 1,0 & 83,0 & - \\
$0,35-0,45$ & 17,0 & 1,0 & 82,0 & - \\
$0,45-0,55$ & 18,0 & 1,0 & 81,0 & - \\
$0,55-0,65$ & 18,0 & 1,0 & 81,0 & - \\
\hline
\end{tabular}

O conjunto das variáveis meteorológicas diárias de entrada utilizados pelo modelo, correspondente aos valores diários de temperatura máxima e mínima do ar $\left({ }^{\circ} \mathrm{C}\right)$, precipitação pluviométrica $(\mathrm{mm})$ e radiação solar (MJ $\mathrm{m}^{-2} \mathrm{dia}^{-1}$ ), que foram cedidos pelo IAPAR. Os anos utilizados foram: 1995 a 2009.

O modelo CANEGRO já dispõe de algumas cultivares cadastradas no seu banco de dados, no entanto, segundo estudo desenvolvido por Nassif et al. (2009), O modelo CANEGRO/DSSAT, desenvolvido para cultivares sulafricanas, necessita de parametrização para as cultivares brasileiras. A cultivar utilizada na simulação foi a RB83-2847, cuja calibração foi feita e já vem sendo utilizada em outros trabalhos por Marin et al. $(2009,2010)$.
Para da calibração da cultivar, Marin et al. (2011) utilizou os dados coletados por Suguitani (2006), cujo experimento foi conduzido no Centro de Tecnologia Canavieira (CTC), em Piracicaba (SP), e solo caracterizado como Latossolo Vermelho Amarelo distrófico. A cultivar foi plantada em espaçamento 1,40 $\mathrm{m}$ em sistema irrigado e sequeiro. A análise biométrica envolveu a medida da altura das plantas, perfilhamento, dinâmica foliar, área foliar, e a fitomassa da parte aérea. Mais detalhes sobre o experimento de campo podem ser obtidos em Suguitani (2006).

Os indicadores estatísticos da comparação entre dados medidos e observados do modelo CANEGRO/DSSAT podem ser vistos na Tabela 13, retirada de Marin et al. (2011) para a variedade RB83-2847.

Tabela 2 - Indicadores Estatísticos de comparação entre dados medidos e observados do modelo CANEGRO/DSSAT para a cultivar RB83-2847 
PRODUTIVIDADE DA CANA-DE-AÇÚCAR RB83-2847 IRRIGADA EM PARANAVAÍ, PARANÁ

\begin{tabular}{|c|c|c|c|c|c|c|}
\hline \multirow[t]{2}{*}{ Variável } & $\mathrm{R}^{2}$ & $\begin{array}{c}\text { Erro } \\
\text { Médio } \\
\text { Absoluto }\end{array}$ & $\begin{array}{c}\text { Índice } \\
D^{*}\end{array}$ & $\mathrm{R}^{2}$ & $\begin{array}{c}\text { Erro } \\
\text { Médio } \\
\text { Absoluto } \\
\end{array}$ & $\begin{array}{c}\text { Índice } \\
\text { D }\end{array}$ \\
\hline & \multicolumn{3}{|c|}{ Não Irrigado } & \multicolumn{3}{|c|}{ Irrigado } \\
\hline IAF & 1,00 & 1,2 & 0,57 & 1,00 & 0,66 & 0,79 \\
\hline Massa Seca Colmos (t/ha) & 0,99 & 6,74 & 0,90 & 0,99 & 6,18 & 0,91 \\
\hline Massa Seca Aérea (t/ha) & 0,99 & 2,85 & 0,99 & 1,00 & 3,36 & 0,98 \\
\hline Perfilhos $/ \mathrm{m}^{2}$ & 0,75 & 4,01 & 0,3 & 0,77 & 3,5 & 0,39 \\
\hline Altura m & 0,98 & 0,27 & 0,95 & 0,99 & 0,24 & 0,97 \\
\hline
\end{tabular}

(MARIN et al., 2011)

Os cenários utilizados nas simulações foram baseados na época de plantio -4 datas utilizadas: 15 de janeiro (Dia Juliano 15), 15 de março (Dia Juliano 74), 15 de setembro (Dia Juliano 258) e 15 de novembro (Dia Juliano 319); duas épocas de colheita - cana de ano e de ano e meio; e utilização da irrigação - irrigado ou sequeiro. Foram simulados 15 anos de plantio para cada localidade. Assim, para cada localidade foram consideradas 4 datas de plantio, 2 épocas de colheita e o sistema irrigado e não, totalizando 16 cenários por região.

A Irrigação era calculada automaticamente pelo modelo, quando a umidade atingia o nível crítico aplicava-se a lâmina necessária para voltar a capacidade de campo. O método de irrigação foi o aspersão, a profundidade de manejo adotada foi de $0,7 \mathrm{~m}$.

As datas de colheita para os plantios realizados em janeiro eram de 15 de janeiro, para plantio de 1 ano e 15 de julho para plantio de 1,5 anos; para março: 15 de março, para plantio de 1 ano e 15 de setembro para plantio de 1,5 anos; para setembro: 15 de setembro, para plantio de 1 ano e 15 de março para plantio de 1,5 anos; para novembro: 15 de novembro, para plantio de 1 ano e 15 de maio para plantio de 1,5 anos.

As análises de variância das produtividades avaliadas foram analisadas com auxílio do software SISVAR. Para os dados de produtividades utilizou-se o teste de Tukey ao nível de 5\% de probabilidade, e para a renda bruta foi utilizado o teste de
Scott-Knot ao nível de $5 \%$ de probabilidade.

Para facilitar a discussão dos dados serão utilizadas as nomenclaturas: JAN, MAR, SET, NOV, para representar o mês de plantio; SEC e IRR, para representar o plantio de sequeiro ou irrigado; juntamente com 1 ou 5, para representar se o tempo da cultura no campo foi de 1 ano ou 1,5 anos. Assim, por exemplo, SEC1 representará o plantio em sequeiro com duração de um ano.

\section{RESULTADOS E DISCUSSÃO}

$\mathrm{Na}$ Tabela 3 são apresentados as produtividades máximas, mínimas e médias simuladas de produtividade de colmos e açúcar e seus respectivos coeficientes de variação (CV), no município de Paranavaí, em diferentes épocas de plantio e tempo de colheita. Esses valores de produtividade nas condições de simulação são para uma produção potencial.

Nos plantios em sequeiro os coeficientes de variação $(\mathrm{CV})$ variaram de 10,90 a $14,36 \%$ e 8,76 a $11,35 \%$, para produtividade de massa fresca e de 13,58 a $18,28 \%$ e 8,93 a $12,39 \%$ para açúcar, alterando apenas a época de colheita. MAR apresentou a melhor média para SEC1, tanto para produtividade de colmos, quanto para açúcar. Para 1,5 anos, as melhores médias foram para SET.

Em IRR1 ocorre redução dos valores de CV - colmo e CV - açúcar, em relação ao SEC1, acompanhada de um aumento de produtividade de colmo e de açúcar, no entanto, a redução de CV - 
açúcar só foi menor em NOV, comparado ao SEC1.5, e, comparada, ainda ao sistema SEC1.5, IRR1 só apresentou produtividade média superior em MAR, para colmos; para açúcar, nenhum mês obteve produtividade média superior. Mostrando, mais uma vez, que a influência na produtividade da cana-de-açúcar é mais sentida pelo aumento da época da cultura no campo do que com o uso da técnica da irrigação.

Tabela 3 - Produtividade de colmos $\left({\mathrm{Mg} . \mathrm{ha}^{-1}}^{-1}\right)$ e de açúcar (Mg.ha-1) simuladas para as quatro épocas de plantio, sob condições irrigadas e de sequeiro para o município de Paranavaí - PR

\begin{tabular}{|c|c|c|c|c|c|c|c|c|}
\hline & \multicolumn{8}{|c|}{ Paranavaí-PA } \\
\hline & \multicolumn{8}{|c|}{ Mg.Colmos.ha- ${ }^{1}$ para 1 ano de plantio } \\
\hline & \multicolumn{4}{|c|}{ Produtividade Sequeiro } & \multicolumn{4}{|c|}{ Produtividade Irrigada } \\
\hline Plantio & Média & Máxima & Mínima & $\mathrm{CV}(\%)$ & Média & Máxima & Mínima & $\mathrm{CV}(\%)$ \\
\hline JAN & 106,27 & 125,43 & 85,26 & 11,18 & 132,58 & 145,79 & 117,80 & 6,34 \\
\hline MAR & 116,08 & 131,16 & 91,66 & 12,32 & 140,18 & 170,89 & 124,62 & 8,20 \\
\hline SET & 99,35 & 111,38 & 76,96 & 10,90 & 118,02 & 147,25 & 102,32 & 8,67 \\
\hline NOV & 103,92 & 130,73 & 84,38 & 14,36 & 124,32 & 146,17 & 111,18 & 8,22 \\
\hline
\end{tabular}

Mg.Colmos.ha-1 para 1,5 anos de plantio

Produtividade Sequeiro

\begin{tabular}{|c|c|c|c|c|c|c|c|c|}
\hline \multirow[b]{2}{*}{ Plantio } & \multicolumn{4}{|c|}{ Produtividade Sequeiro } & \multicolumn{4}{|c|}{ Produtividade Irrigada } \\
\hline & Média & Máxima & Mínima & $\mathrm{CV}(\%)$ & Média & Máxima & Mínima & $\mathrm{CV}(\%)$ \\
\hline JAN & 136,76 & 157,36 & 116,01 & 8,76 & 158,46 & 192,32 & 142,60 & 7,22 \\
\hline MAR & 131,90 & 147,97 & 110,24 & 8,95 & 154,64 & 194,67 & 142,07 & 7,90 \\
\hline SET & 152,19 & 181,38 & 134,04 & 9,54 & 171,38 & 201,83 & 159,35 & 7,43 \\
\hline NOV & 147,76 & 175,02 & 122,93 & 11,35 & 166,58 & 192,75 & 149,73 & 7,91 \\
\hline
\end{tabular}

Mg.Açúcar.ha-1 ${ }^{1}$ para 1 ano de plantio

Produtividade Sequeiro

\begin{tabular}{|c|c|c|c|c|c|c|c|c|}
\hline \multirow[b]{2}{*}{ Plantio } & \multicolumn{4}{|c|}{ Produtividade Sequeiro } & \multicolumn{4}{|c|}{ Produtividade Irrigada } \\
\hline & Média & Máxima & Mínima & $\mathrm{CV}(\%)$ & Média & Máxima & Mínima & $\mathrm{CV}(\%)$ \\
\hline JAN & 12,57 & 15,44 & 9,46 & 16,54 & 16,57 & 21,56 & 14,27 & 12,79 \\
\hline MAR & 13,08 & 16,02 & 8,93 & 15,96 & 16,80 & 23,07 & 13,53 & 13,22 \\
\hline SET & 12,83 & 14,95 & 9,77 & 13,58 & 15,89 & 21,76 & 13,16 & 12,70 \\
\hline NOV & 12,93 & 17,38 & 9,77 & 18,28 & 16,18 & 19,14 & 13,88 & 9,53 \\
\hline
\end{tabular}

Mg.Açúcar.ha- ${ }^{1}$ para 1,5 anos de plantio

Produtividade Sequeiro

Produtividade Irrigada

\begin{tabular}{|c|c|c|c|c|c|c|c|c|}
\hline \multirow[b]{2}{*}{ Plantio } & & \\
\hline & Média & Máxima & Mínima & $\mathrm{CV}(\%)$ & Média & Máxima & Mínima & $\mathrm{CV}(\%)$ \\
\hline JAN & 17,72 & 20,59 & 15,43 & 8,93 & 20,97 & 28,54 & 18,56 & 11,11 \\
\hline MAR & 17,41 & 21,04 & 14,98 & 8,95 & 20,96 & 29,63 & 18,50 & 12,28 \\
\hline SET & 20,04 & 23,52 & 16,84 & 9,69 & 23,32 & 28,73 & 19,56 & 9,25 \\
\hline NOV & 19,51 & 23,61 & 16,51 & 12,39 & 22,57 & 27,81 & 19,33 & 9,72 \\
\hline
\end{tabular}

A utilização da irrigação junto com o aumento do ciclo proporcionou aumento de produtividade e redução do coeficiente de variação em relação à produtividade SEC1, SEC1.5 e IRR1, tanto para produtividade açúcar, quanto para colmo, e sua produtividade média foi superior a qualquer outro cenário. Demonstrando que na cidade estudada, a interação época de plantio x irrigação foi benéfica. Barros et al. (2012) observou em seu estudo com as cidades de Gurupi-To e Teresina-PI não foi benéfica.

As produtividades máximas alcançadas foram encontradas em: MAR, para colmo SEC1 e IRR1, para açúcar: IRR1 e IRR1.5; SET, colmo: SEC1.5 e IRR1.5; NOV, para açúcar: SEC1 e SEC1.5. As mínimas foram em: MAR, para colmo SEC1.5 e IRR1.5, para açúcar SEC1, SEC1.5 e IRR1.5; e SET, para colmo SEC1 e IRR1, açúcar IRR1. Existe uma maior variação entre os meses que 
possuem as maiores produtividades, e dessa vez, o MAR possuiu a maior quantidade de produtividade em diferentes cenários e JAN não apresenta nenhum das menores produtividades.

Oliveira, et al. (2007) avaliou a produtividade da cana-de-açúcar na região de Paranavaí, em sistema de sequeiro no mesmo tipo de solo do utilizado nesse trabalho, com o plantio realizado em março e colheita 377 DAP, utilizando a cultivar RB85-5113 e obteve produtividade de 116,5 Mg.ha- ${ }^{1}$, valor quase idêntico ao encontrado para a média dos plantios realizados em março no sistema de SEC1 (sequeiro, com colheita um ano depois).

Os mesmos autores realizaram a colheita do experimento em outras duas datas e obtiveram as produtividades de 131,7 Mg.ha- ${ }^{1}$ e 133,1 Mg.ha- ${ }^{1}$, para 428 DAP e 497 DAP, valores também quase idênticos aos encontrados para os plantios em janeiro e, principalmente, março. Mostrando a grande precisão do modelo para regiões com boa disponibilidade hídrica, quando se produz a cana em esquema de sequeiro.

Para a discussão dos dados mais minuciosa foi feita a estatística dos mesmos, nessa estatística foi avaliada a interação entre os manejos (SEC1, IRR $1, \ldots$ ) dentro de cada mês e a interação dos meses dentro de cada manejo (Tabela 4Erro! Fonte de referência não encontrada.). Diferentemente do que foi visto nas outras regiões podemos notar que na produtividade de colmos houve diferenciação estatística nos meses que produziram sob irrigação.

$\mathrm{Na}$ produtividade de açúcar, os manejos SEC1 e IRR1 não apresentaram diferenciação, dentro dos meses.

Tabela 4 - Análise estatística dos dados de produtividade média em relação aos meses dentro dos manejos adotado e dos manejos dentro de cada mês de plantio para a cidade de Paranavaí - PR

\begin{tabular}{|c|c|c|c|c|}
\hline \multicolumn{5}{|c|}{ Teresina-PI } \\
\hline \multirow[b]{3}{*}{ meses } & \multicolumn{4}{|c|}{ Manejo } \\
\hline & SEC1 & IRR1 & SEC1.5 & IRR1.5 \\
\hline & \multicolumn{4}{|c|}{ Mg.Colmos.ha-1 } \\
\hline JAN & $106,27 \mathrm{a}$ & $132,58 \mathrm{bc}$ & $136,76 \mathrm{ab}$ & 158,46 a \\
\hline MAR & $116,08 \mathrm{ab}$ & $140,18 \mathrm{a}$ & $131,90 \mathrm{a}$ & 154,64 a \\
\hline SET & $99,35 \mathrm{a}$ & $118,02 \mathrm{a}$ & $152,19 \mathrm{c}$ & $171,38 \mathrm{~b}$ \\
\hline \multirow[t]{3}{*}{ NOV } & $103,92 \mathrm{ab}$ & $124,32 \mathrm{ab}$ & $147,76 \mathrm{bc}$ & $166,58 \mathrm{ab}$ \\
\hline & JAN & MAR & SET & NOV \\
\hline & \multicolumn{4}{|c|}{ Mg,Colmos,ha- ${ }^{1}$} \\
\hline SEC1 & $106,27 \mathrm{a}$ & $116,08 \mathrm{a}$ & $99,35 \mathrm{a}$ & $103,92 \mathrm{a}$ \\
\hline IRR1 & $132,58 \mathrm{~b}$ & $140,18 \mathrm{~b}$ & $118,02 \mathrm{~b}$ & $124,32 \mathrm{~b}$ \\
\hline SEC1.5 & $136,76 \mathrm{~b}$ & $131,9 \mathrm{~b}$ & $152,19 \mathrm{c}$ & $147,76 \mathrm{c}$ \\
\hline \multirow[t]{3}{*}{ IRR1.5 } & $158,46 \mathrm{c}$ & $154,64 \mathrm{c}$ & $171,38 \mathrm{~d}$ & $166,58 \mathrm{~d}$ \\
\hline & SEC1 & IRR1 & SEC1.5 & IRR1.5 \\
\hline & \multicolumn{4}{|c|}{ Mg,Açúcar,ha-1 } \\
\hline JAN & $12,57 \mathrm{a}$ & $16,57 \mathrm{a}$ & $17,72 \mathrm{ab}$ & $20,97 \mathrm{a}$ \\
\hline MAR & $13,08 \mathrm{a}$ & $16,80 \mathrm{a}$ & $17,41 \mathrm{a}$ & $20,96 \mathrm{a}$ \\
\hline SET & $12,83 \mathrm{a}$ & 15,89 a & $20,04 \mathrm{c}$ & $23,32 \mathrm{~b}$ \\
\hline \multirow[t]{3}{*}{ NOV } & $12,93 \mathrm{a}$ & $16,18 \mathrm{a}$ & $19,51 \mathrm{bc}$ & $22,57 \mathrm{ab}$ \\
\hline & JAN & MAR & SET & NOV \\
\hline & \multicolumn{4}{|c|}{ Mg,Açúcar,ha-1 } \\
\hline SEC1 & $12,57 \mathrm{a}$ & $13,08 \mathrm{a}$ & $12,83 \mathrm{a}$ & $12,93 \mathrm{a}$ \\
\hline IRR1 & $16,57 \mathrm{~b}$ & $16,8 \mathrm{~b}$ & $15,89 \mathrm{~b}$ & $16,18 \mathrm{~b}$ \\
\hline SEC1.5 & $17,72 \mathrm{~b}$ & $17,41 \mathrm{~b}$ & $20,04 \mathrm{c}$ & $19,51 \mathrm{c}$ \\
\hline IRR1.5 & $20,97 \mathrm{c}$ & $20,96 \mathrm{c}$ & $23,32 \mathrm{~d}$ & $22,57 \mathrm{~d}$ \\
\hline
\end{tabular}


Médias seguidas de mesma letra não diferem entre si pelo teste de Tukey a 5\% probabilidade

5). A utilização da irrigação, aumento do ciclo e a utilização das duas técnicas juntas possibilitaram aumento de produtividade.

A sensibilidade do aumento de açúcar foi maior que o de colmo (Tabela

Tabela 5 - Aumento na produtividade de colmo e açúcar (\%) devido ao incremento da irrigação e/ou do aumento do ciclo, para Paranavaí - PR

\begin{tabular}{|c|c|c|c|c|c|c|}
\hline \multirow[b]{2}{*}{ Plantio } & \multicolumn{2}{|c|}{ Irrigação } & \multicolumn{2}{|c|}{$\begin{array}{l}\text { Aumento do tempo } \\
\text { da cultura no campo }\end{array}$} & \multicolumn{2}{|c|}{$\begin{array}{c}\text { Irrigação e Aumento } \\
\text { do tempo }\end{array}$} \\
\hline & $\begin{array}{c}\text { Colmos } \\
(\%)\end{array}$ & $\begin{array}{l}\text { Açúcar } \\
(\%)\end{array}$ & $\begin{array}{c}\text { Colmos } \\
(\%)\end{array}$ & $\begin{array}{l}\text { Açúcar } \\
(\%)\end{array}$ & $\begin{array}{c}\text { Colmos } \\
(\%)\end{array}$ & $\begin{array}{l}\text { Açúcar } \\
(\%)\end{array}$ \\
\hline JAN & 24,76 & 31,77 & 28,69 & 40,92 & 49,11 & 66,77 \\
\hline MAR & 20,76 & 28,48 & 13,63 & 33,10 & 33,22 & 60,23 \\
\hline SET & 18,79 & 23,83 & 53,18 & 56,24 & 72,50 & 81,77 \\
\hline NOV & 19,63 & 25,10 & 42,19 & 50,86 & 60,30 & 74,50 \\
\hline
\end{tabular}

O maior incremento de produtividade de colmo e açúcar foi devido ao aumento da cultura no campo do que a utilização somente da irrigação, para todos os meses analisados, sendo SET o que apresentou os melhores incrementos. Mostrando que a cana-de-açúcar produzida na cidade de Paranavaí responderia bem melhor ao aumento da cultura no campo do que a irrigação. Apesar da baixa influência da irrigação na produtividade de colmos seu incremento variou de 18,79 a $24,78 \%$, para colmo e 23,83 a $31,77 \%$ para açúcar, e SET foi o menos sensível.

$\mathrm{O}$ incremento de produtividade devido à irrigação $\mathrm{x}$ aumento da época no campo foi o que apresentou os melhores valores.

\section{Análise Econômica}

Para a realização de uma análise econômica mais detalhada seria necessária uma série de outros fatores para chegar a uma conclusão, tais como: custo da terra, custo da água, custo do sistema de irrigação, custo da mão-de-obra, dentre outros, no entanto, a análise da renda mensal bruta, juntamente ao número de irrigações e quantidade de lâminas aplicadas serve para dar uma noção do aspecto econômico da região e por esse motivo são apresentados os dados a seguir.

Considerando o preço de venda do açúcar de $\mathrm{R} \$ 45,00$ a tonelada, foram confeccionados os gráficos a seguir (Figura 1Figura ). Nota-se que o sistema IRR1 é o que apresenta os maiores valores, mostrando uma maior renda em função das técnicas empregadas. Diferentemente do que foi visto nas cidades discutidas anteriormente, em Paranavaí, ocorre menor diferença entre o sistema irrigado e de sequeiro.

A utilização do sistema SEC1.5 é uma das mais desvantajosas técnicas para aumentar a produtividade em relação a SEC1, já que para JAN e MAR ocorre redução da renda, para NOV o aumento é menor que $1 \%$ e SET não foi superior a $4,5 \%$. A variação entre os meses de plantio é menor para SEC1. 


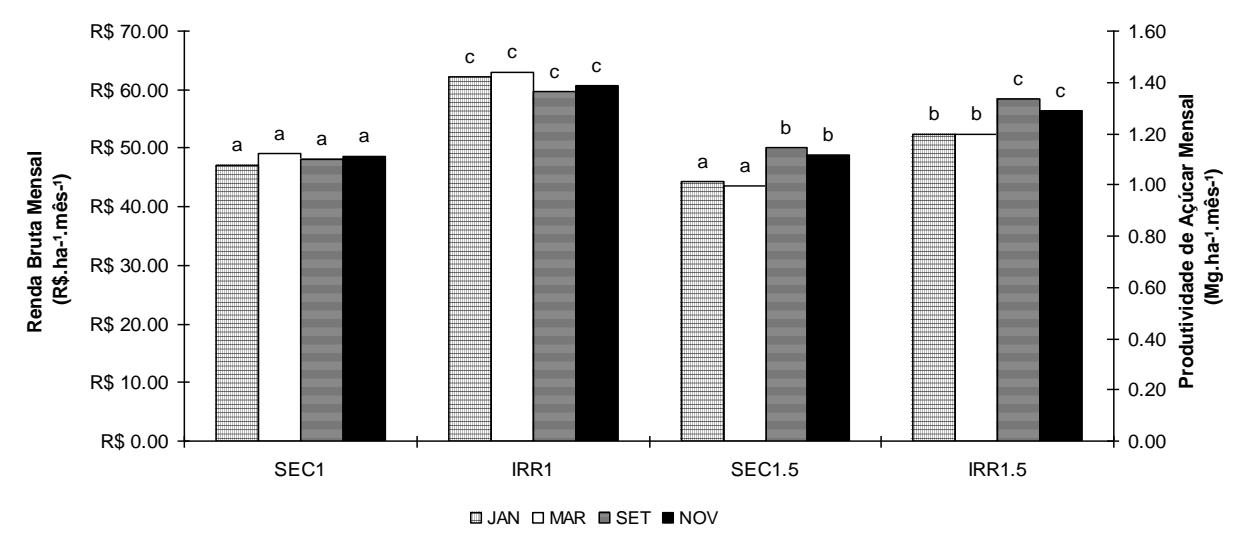

Figura 1 - Renda bruta pelo número de meses da cultura no campo da produtividade de açúcar (R\$) para cada cenário avaliado, nas cidades de Paranavaí

\section{CONCLUSÃO}

O modelo apresentou ser útil na simulação da cana-de-açúcar na região de Paranavaí-PR.

A produtividade atingida para a cultivar RB83-2847 em Paranavaí foi entre 99 e 171 Mg.ha-1.

\section{REFERÊNCIAS}

ANDRADE JÚNIOR, A.S.; DUARTE, J. A. L.; MOTA, P. R. D. ; MOURA, J. F. L.; RIBEIRO, V. Q. Níveis de irrigação e fertirrigação por gotejamento subsuperficial na produção de cana-deaçúcar. In: CONGRESSO BRASILEIRO DE ENGENHARIA AGRÍCOLA, 38., 2009, Juazeiro; Petrolina. Anais... Juazeiro; Petrolina: SBEA; UNIVASF, 2009. 1 CD-ROM.

BARROS, A. C.; COELHO, R. D.; MARIN, F. R.; POLZER, D. L.; AGUIAR NETTO, A. O. Utilização do modelo CANEGRO para estimativa de crescimento da cana-de-açúcar irrigada e não irrigada para as regiões de Gurupi TO e Teresina - PI. Irriga, Botucatu. 2012. (aceito para publicação em 2012).

CANA-DE-AÇÚCAR. Agrianual 2009: Anuário estatístico da agricultura brasileira. São Paulo: FNP, p. 249, 2009.
Baseada numa análise simplificada a irrigação não é economicamente viável em Paranavaí.

\section{AGRADECIMENTOS}

Ao Iapar pelo fornecimento dos dados climatológicos.

CAPUTO, M. M. et al. Respostas de genótipos de cana-de-açúcar à aplicação de indutores de maturação. Bragantia, v. 67, n. 1, p. 15-23, 2008.

CHEEROO-NAYAMUTH, $\quad$ F.C.; ROBERTSON, M.J.; WEGENER, M.K.; NAYAMUTH, A.RH. Using a simulation model to assess potential and attainable sugar cane yield in Mauritius. Field Crops Research, Amsterdam, v.66, p.225-243, 2000.

DARLI, A. B.; Raimundo, L. C.; Garcia, C. J. B.; Duenhas, L. H. Irrigação por gotejamento subsuperficial na produção e qualidade de cana-de-açúcar. Irriga, Botucatu, v. 13, n. 1, p. 1-11, 2008.

ESTUDO de sustentabilidade da produção de etanol de cana-de-cçúcar. Subsídios Técnicos para a Agenda Brasileira de Bioetanol. Relatório final. Brasília, DF, Centro de Gestão e Estudos Estratégicos, 2009. 337 p. 
GODOY, A. P. Modelagem de processos de acumulação de biomassa e de açúcar da cana-de-açúcar via sistemas nebulosos. 2007. Dissertação (Mestrado em Engenharia Elétrica) - Faculdade de Engenharia Elétrica e Computação, Universidade Estadual de Campinas, Campinas, 2007.

INMAN-BAMBER, N.G. CANEGRO: its history, conceptual basis, present and future uses. In: Robertson, M.J. (Ed.) Research and modelling approaches to assess sugarcane production opportunities and constraints. University of Queensland, Brisbane, 1994. p. 31-34.

MARIN, F. R.; JONES, J. W.; ROYCE, F.; SUGUITANI, C.; DONZELI, J. L.; PALLONE FILHO, W. J.; NASSIF, D. S. P. Parameterization and evaluation of predictions of DSSAT/CANEGRO for sugarcane Brazilian productions systems. Agronomy Journal, Madison, v. 103, p. 100-110, 2011.

MARIN, F.R.; JONES, J. W.; ROYCE, F.; SUGUITANI, C.; DONZELI, J.L.; PALLONE FILHO, W.J.; NASSIF, D.S.P. Parameterization and evaluation of predictions of DSSAT/CANEGRO for sugarcane Brazilian productions systems. Agronomy Journal, Madison, v. 103, p. 100-110, 2011.

MARUN, F. Propriedades físicas e biológicas de um latossolo vermelhoescuro do arenito caiuá sob pastagem e culturas anuais. Pesquisa Agropecuária Brasileira, Brasília, v. 31, n. 8, p. 593597, 1996.

NASSIF, D. S. P. Parametrização e avaliação do modelo DSSAT/CANEGRO para variedades brasileiras de cana-de-açúcar. 2011. Dissertação de Mestrado (Mestrado em Física do Ambiente Agrícola) - Escola Superior de Agricultura "Luiz de Queiroz",
Universidade de São Paulo, Piracicaba, 2011.

O'LEARY, G. J. Modeling sugarcane production systems i. development and performance of sugarcane module. Field Crops Research, Amsterdam, v. 61, p. 253-271, 1999.

OLIVEIRA, R.A.; DAROS, E.C.; ZAMBON, J.L.C.; WEBER, H.; IDO, O.T., ESPALHOK-FILHO, J.C.; RIBAS, K.C.Z.; SILVA, D.K.T. Área foliar em três cultivares de cana-de-açúcar e sua correlação com a produção de biomassa. Ver. Agrop. Trop. 37 (2): 71-76, 2007.

RAMOS, F. A. P. Comportamento da cana-de-açúcar cultivar $S p$ 791011, submetidas a diferentes épocas de plantio em duas condições edafoclimáticas. 2006. 50 f. Dissertação (Pós-graduação em Manejo de Solo e Água) - Universidade Federal da Paraíba, Areia, 2006.

SCARPARI, M.S. PREDPOL: um modelo de previsão da maturação da cana-de-açúcar visando planejamento otimizado. 2007. 120 p. (Doutorado em Fitotecnia) - Escola Superior de Agricultura "Luiz de Queiroz", Universidade de São Paulo, Piracicaba, 2007.

SILVA, C. R.; ANDRADE JÚNIOR, A. S.; ALVES JÚNIOR, J.; SOUZA, A. B.; MELO, F. B.; COELHO FILHO, M. C. Calibration of a capacitance probe In a paleudult. Scientia Agricola. Piracicaba, v. 64, n. 6, p. 636-640, 2007.

SUGUITANI, C. Fenologia da cana-deaçúcar (Saccharum spp.) sob efeito do fósforo. 2001. 79 p. Dissertação (Mestrado em Fitotecnia) - Escola Superior de Agricultura "Luiz de Queiroz", Universidade de São Paulo, Piracicaba, 2001. 